MATHEMATICS OF COMPUTATION

Volume 80, Number 276, October 2011, Pages 2359-2379

S $0025-5718(2011) 02467-1$

Article electronically published on February 17, 2011

\title{
CALCULATING CYCLOTOMIC POLYNOMIALS
}

\author{
ANDREW ARNOLD AND MICHAEL MONAGAN
}

\begin{abstract}
We present three algorithms to calculate $\Phi_{n}(z)$, the $n_{t h}$ cyclotomic polynomial. The first algorithm calculates $\Phi_{n}(z)$ by a series of polynomial divisions, which we perform using the fast Fourier transform. The second algorithm calculates $\Phi_{n}(z)$ as a quotient of products of sparse power series. These two algorithms, described in detail in the paper, were used to calculate cyclotomic polynomials of large height and length. In particular, we have found the least $n$ for which the height of $\Phi_{n}(z)$ is greater than $n, n^{2}, n^{3}$, and $n^{4}$, respectively. The third algorithm, the big prime algorithm, generates the terms of $\Phi_{n}(z)$ sequentially, in a manner which reduces the memory cost. We use the big prime algorithm to find the minimal known height of cyclotomic polynomials of order five. We include these results as well as other examples of cyclotomic polynomials of unusually large height, and bounds on the coefficient of the term of degree $k$ for all cyclotomic polynomials.
\end{abstract}

\section{INTRODUCTION}

The $n_{t h}$ cyclotomic polynomial, $\Phi_{n}(z)$, is the monic polynomial whose $\phi(n)$ distinct roots are exactly the $n_{t h}$ primitive roots of unity.

$$
\Phi_{n}(z)=\prod_{\substack{j=1 \\ g c d(j, n)=1}}^{n}\left(z-e^{2 \pi i j / n}\right) .
$$

It is an irreducible polynomial over $\mathbb{Z}$ with degree $\phi(n)$, where $\phi(n)$ is Euler's totient function. The $n_{t h}$ inverse cyclotomic polynomial, $\Psi_{n}(z)$, is the polynomial whose roots are the $n_{t h}$ nonprimitive roots of unity.

$$
\Psi_{n}(z)=\prod_{\substack{j=1 \\ g c d(j, n)>1}}^{n}\left(z-e^{2 \pi i j / n}\right) .
$$

As the roots of $\Phi_{n}(z)$ and $\Psi_{n}(z)$ comprise all $n_{t h}$ roots of unity, we have

$$
\Psi_{n}(z)=\frac{z^{n}-1}{\Phi_{n}(z)} .
$$

For more about inverse cyclotomic polynomials, see Moree [19].

Let the order of $\Phi_{n}(z)$ denote the number of distinct odd prime divisors of $n$. To make the distinction between the order of $\Phi_{n}(z)$ and $n$, we will refer to $n$ as the index of $\Phi_{n}(z)$ in this paper.

Received by the editor October 10, 2008 and, in revised form, July 17, 2010.

2010 Mathematics Subject Classification. Primary 11Y16, Secondary 12-04.

This work was supported by NSERC of Canada and the MITACS NCE of Canada.

(C)2011 American Mathematical Society Reverts to public domain 28 years from publication 
We write

$$
\Phi_{n}(z)=\sum_{k=0}^{\phi(n)} a_{n}(k) z^{k} \quad \text { and } \quad \Psi_{n}(z)=\sum_{k=0}^{n-\phi(n)} c_{n}(k) z^{k},
$$

and define $a_{n}(k)=0$ for $k>\phi(n)$. We let $A(n)$ and $S(n)$ be the height and length, respectively, of $\Phi_{n}(z)$. That is,

$$
A(n)=\left\|\Phi_{n}(z)\right\|_{\infty}=\max _{0 \leq k \leq \phi(n)}\left|a_{n}(k)\right| \quad \text { and } \quad S(n)=\left\|\Phi_{n}(z)\right\|_{1}=\sum_{k=0}^{\phi(n)}\left|a_{n}(k)\right| .
$$

For $n<105$, all the coefficients of $\Phi_{n}(z)$ are $-1,0$, or 1 ; however, for $n=105$ we find that $A(105)=2$. Paul Erdös 7 proved that $A(n)$ is not bounded above by any polynomial in $n$. That is, for any constant $c>0$, there exists $n$ such that $A(n)>n^{c}$. There is a wealth of material on the behaviour of $A(n)$ and the size of cyclotomic polynomial coefficients [4, 5, 17, 25]; however, computation may yet provide more insight. Koshiba [15, 16] calculated $A(4849845)=669606$ and found the coefficients of $\Phi_{n}(z)$ with degree less than $\phi(n) / 10$ for $n=111546435=3 \cdot 5 \cdot 7 \cdot 11 \cdot 13 \cdot 17 \cdot 19 \cdot 23$ and, in particular, to our knowledge, the first computed cyclotomic coefficient $a_{n}(k)$ such that $a_{n}(k)>n$. T.D. Noe has made a wealth of data about cyclotomic polynomials and their coefficients available at the Sloane On-Line Encyclopedia of Integer Sequences (see [20, 21, 22, 23, 24], for instance).

A cyclotomic polynomial $\Phi_{n}(z)$ is said to be flat if $A(n)=1$. We say that $\Phi_{n}(z)$ is flatter than $\Phi_{m}(z)$ if $A(m)<A(n)$. It is currently unknown whether there are flat cyclotomic polynomials of order five or greater. We find many examples of flat cyclotomic polynomials of order three and four, and many examples of $\Phi_{n}(z)$ of order five and height 2.

A related problem of interest is the behaviour of the $z^{k}$ coefficient $a_{n}(k)$ of $\Phi_{n}(z)$ over $n \in \mathbb{N}$. To that end let $a(k)=\max _{n}\left|a_{n}(k)\right|$. Bachman [1] developed an asymptotic formula for $\log a(k)$ that improved on a result of Montgomery and Vaughan [18. Noe 20] computed $a(k)$ for $k \leq 1000$. We independently verify $a(k)$ for $k \leq 172$ by way of a brute-force approach. We also extend results of Bosma [6], who computed the least value of $k$ for which $b \in \mathbb{Z}$ occurs as $a_{n}(k)$ for some $n$, for $|b| \leq 50$.

Our motivation for developing algorithms to calculate cyclotomic polynomials was to further study the coefficients of $\Phi_{n}(z)$. In particular, we were interested in studying $A(n)$. We found, for instance, that $n=1181895$ is the smallest $n$ for which $A(n)>n$. Until recently, it was impractical to use modern computer algebra systems to calculate $\Phi_{n}(z)$ for $n$ in the millions. With the development and implementation of the algorithms in this paper, we are able to compute $\Phi_{n}(z)$ for $n$ in the billions and, for some specific, nontrivial cases, well beyond that.

1.1. Organization of paper. We present three algorithms to calculate cyclotomic polynomials. Our first algorithm calculates $\Phi_{n}(z)$ for squarefree $n$ via a series of polynomial divisions. We use the discrete fast Fourier transform to perform these divisions quickly. The second algorithm calculates $\Phi_{n}(z)$ as a quotient of products of sparse power series. The third algorithm outputs the coefficients of $\Phi_{n}(z)$ sequentially, in a manner which requires less memory than it takes to store $\Phi_{n}(z)$, a limitation of the first two algorithms. 
Using these three algorithms, we have produced a library of data on the coefficients of cyclotomic polynomials. Amongst our results, we have computed:

[I] $A(n)$ and $S(n)$ for odd, squarefree $n$ amongst the following:

- $n<10^{8}$ with three prime factors.

- $n<3 \cdot 10^{8}$ with four prime factors.

- $n<6.5 \cdot 10^{8}$ with five prime factors.

- $n<10^{9}$ with six prime factors.

- $n<2.36 \cdot 10^{9}$ with seven prime factors.

- $n<5.6 \cdot 10^{9}$ with eight prime factors.

- $n<1.50 \cdot 10^{10}$ with nine prime factors.

[II] The height and length of $\Psi_{n}(z)$ for odd, squarefree $n$ amongst the following:

- $n<10^{8}$ with three prime factors.

- $n<10^{8}$ with four prime factors.

- $n<5 \cdot 10^{8}$ with five prime factors.

- $n<5 \cdot 10^{8}$ with six prime factors.

- $n<10^{9}$ with seven prime factors.

- $n<2 \cdot 10^{9}$ with eight prime factors.

[III] The least $n$ for which $A(n)>n, n^{2}, n^{3}, n^{4}$, respectively, and other values of unusually large height (Table 6 and Table 7 ).

[IV] The least $n$ such that $A(n)>2^{64}$ (Table 6 ).

[V] $\max _{n} a_{n}(k), \min _{n} a_{n}(k)$, and $a(k)=\max _{n}\left|a_{n}(k)\right|$ for $k \leq 172$ (partially verifying a result of Noe [20]).

[VI] The least integer $k$ for which, given $b$, there exists $n$ such that $a_{n}(k)=b$ for $|b| \leq 927$ (Table 10).

[VII] Many examples of $\Phi_{n}(z)$ of order 5 with height 2 (Table 9).

We include some of the more noteworthy results in this paper; however, all of the data listed above is available on the web at

http://www.cecm.sfu.ca/ ada26/cyclotomic/

1.2. Preliminaries. We are interested in computing cyclotomic polynomials of large height. The following two identities are useful:

Lemma 1. Let $n>1$ be odd. Then $\Phi_{2 n}(z)=\Phi_{n}(-z)$.

Lemma 2. Let $p$ be a prime that divides $n$. Then $\Phi_{n p}(z)=\Phi_{n}\left(z^{p}\right)$.

Lemma 1 tells us that $A(2 n)=A(n)$ and $S(2 n)=S(n)$ for odd $n$. Lemma 2 implies that $A(n p)=A(n)$ and $S(n p)=S(n)$ for $p$ dividing $n$. For the remainder of the paper, we will be strictly concerned with the calculation of cyclotomic polynomials of squarefree, odd index. Lemmas 11 and 2 provide an easy means of calculating $\Phi_{n}(z)$ for $n$ even or nonsquarefree, provided we can calculate $\Phi_{m}(z)$, where $m$ is the greatest squarefree, odd divisor of $n$.

\section{Calculating $\Phi_{n}(z)$ as a polynomial quotient VIA The FAST Fourier Transform}

Our first algorithm calculates $\Phi_{n}(z)$ by a series of polynomial divisions. Our algorithm uses the following identity, which is easy to verify by showing that both sides have the same roots.

Lemma 3. Let $p$ be a prime that does not divide $m$. Then $\Phi_{m p}(z)=\frac{\Phi_{m}\left(z^{p}\right)}{\Phi_{m}(z)}$. 
We are thus able to calculate $\Phi_{n}(z)$, for $n$ of the form

$$
n=p_{1} p_{2} \cdots p_{k}=m p,
$$

with largest prime divisor $p=p_{k}$, by repeated polynomial division, as detailed in Algorithm 1

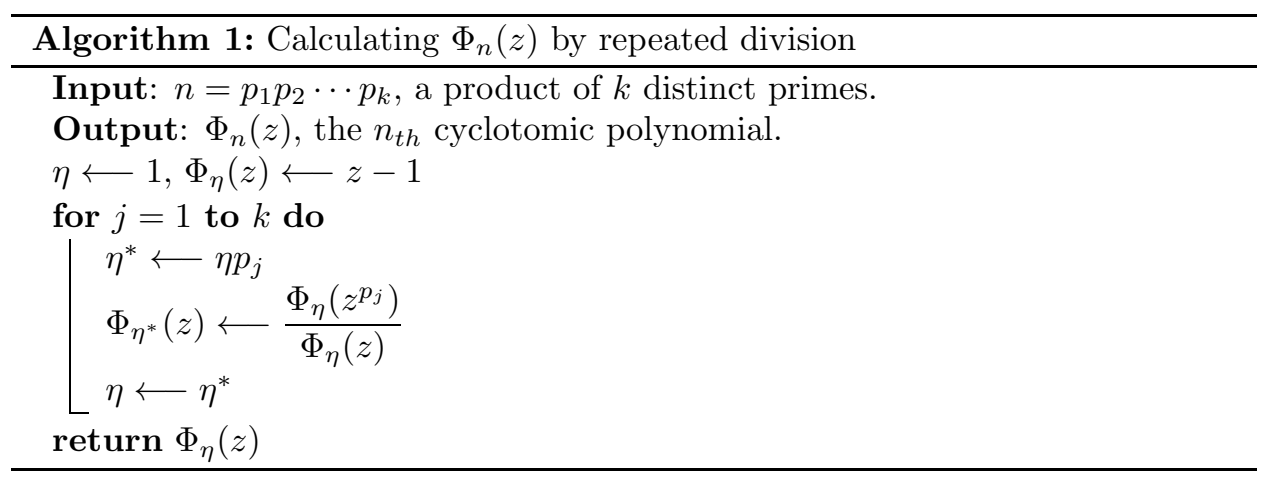

This algorithm (see [26) is well known. It is used in many computer algebra systems. For example, in Maple 13 and in prior versions, it is used by the numtheory[cyclotomic] command. Using classical, quadratic polynomial division, however, is much too slow to do any extensive search on cyclotomic polynomials of index $n$ over one million. For example, Maple 13 takes over five minutes to find $\Phi_{255255}(z)$ (see Table 3 for timings). We implemented Algorithm 1 with machine-precision arithmetic and optimized the polynomial division in Algorithm 1 by way of the discrete Fast Fourier Transform (FFT) 9, 26]. Our implementation of Algorithm 1 can calculate $\Phi_{255255}(z)$ modulo two 32-bit primes in under one second. Algorithm 2 gives a high-level description of the division calculation via the FFT.

Using the Fast Fourier Transform, we can calculate $\Phi_{n}(z)=\frac{\Phi_{m}\left(z^{p}\right)}{\Phi_{m}(z)}$ modulo a prime $q$ in $\mathcal{O}(N \log (N))$ arithmetic operations, where $N$ is the smallest power of 2 greater than $\phi(n)$, the degree of the quotient. We need not interpolate using $N>\phi(m) p$, the degree of the numerator, as we know division of $\Phi_{m}\left(z^{p}\right)$ by $\Phi_{m}(z)$ is exact.

Observe that Algorithm2 2 requires that $B_{i}$ is nonzero modulo $q$ for $\omega^{i}, 0 \leq i<N$. This does not pose a problem for odd indices $m$, however, by the following lemma.

Lemma 4. Let $m>1$ be an odd, squarefree integer, and let $p, N, q$, and $\omega$ be as defined in Algorithm 2. Then $\Phi_{m}\left(\omega^{i}\right) \neq 0 \bmod q$ for $0 \leq i<N$.

Proof. Every power of $\omega$ is a $\left(2^{k}\right)_{t h}$ root of unity modulo $q$ for some $k \leq s$. For $m>1$ it holds that $\Phi_{m}(z)$ divides

$$
\frac{z^{m}-1}{z-1}=z^{m-1}+z^{m-1}+\cdots+z+1 .
$$

Thus as $q$ does not divide $m$, any root of $\Phi_{m}(z) \bmod q$ is necessarily an $m_{t h}$ root of unity not equal to 1 . Given $m$ is odd for our purposes, the only $m_{t h}$ root of unity that is also a $\left(2^{k}\right)_{t h}$ root of unity modulo $q$ for some $k$ is exactly 1 , and so $\Phi_{m}\left(\omega^{i}\right) \neq 0 \bmod q$ for $0 \leq i<N$. 


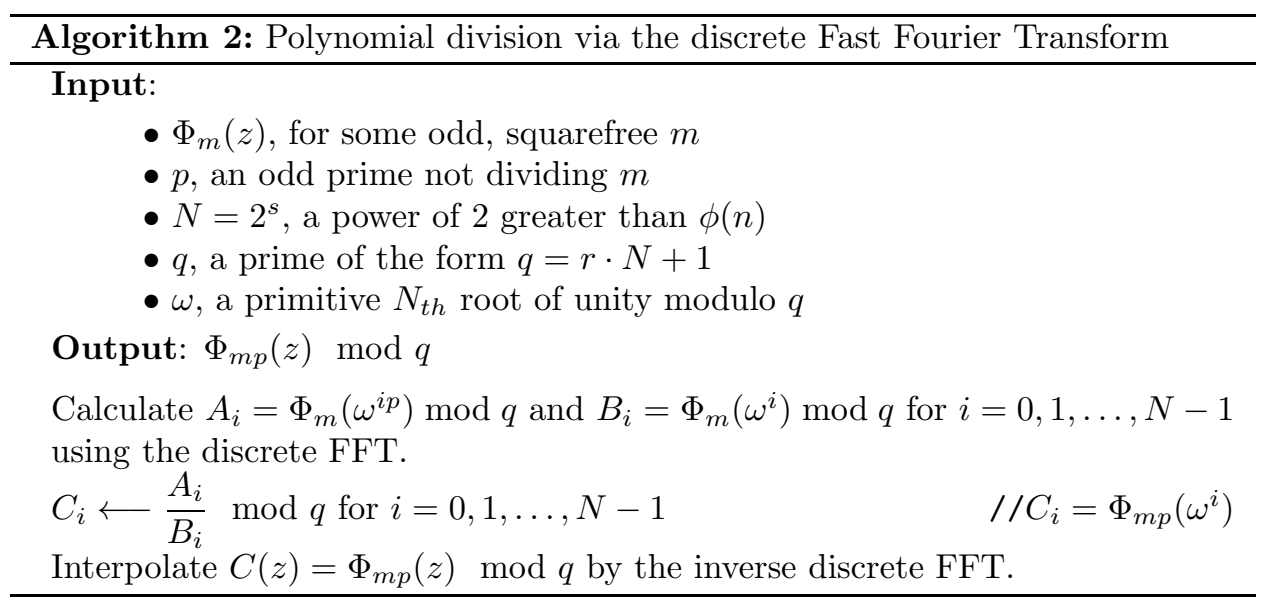

2.1. Implementation details. For primes $q<2^{32}$ of the form $q=r N+1, N$ cannot exceed $2^{27}$. Thus for cyclotomic polynomials of large degree, we require primes larger than $2^{32}$. Choosing unnecessarily large primes for the FFT would require multiprecision arithmetic. Using 64-bit arithmetic, we are able to multiply modulo prime numbers as large as 42-bits (Algorithm 3); however, multiplication modulo a 42-bit prime is roughly twice as slow as multiplication modulo a 32-bit prime.

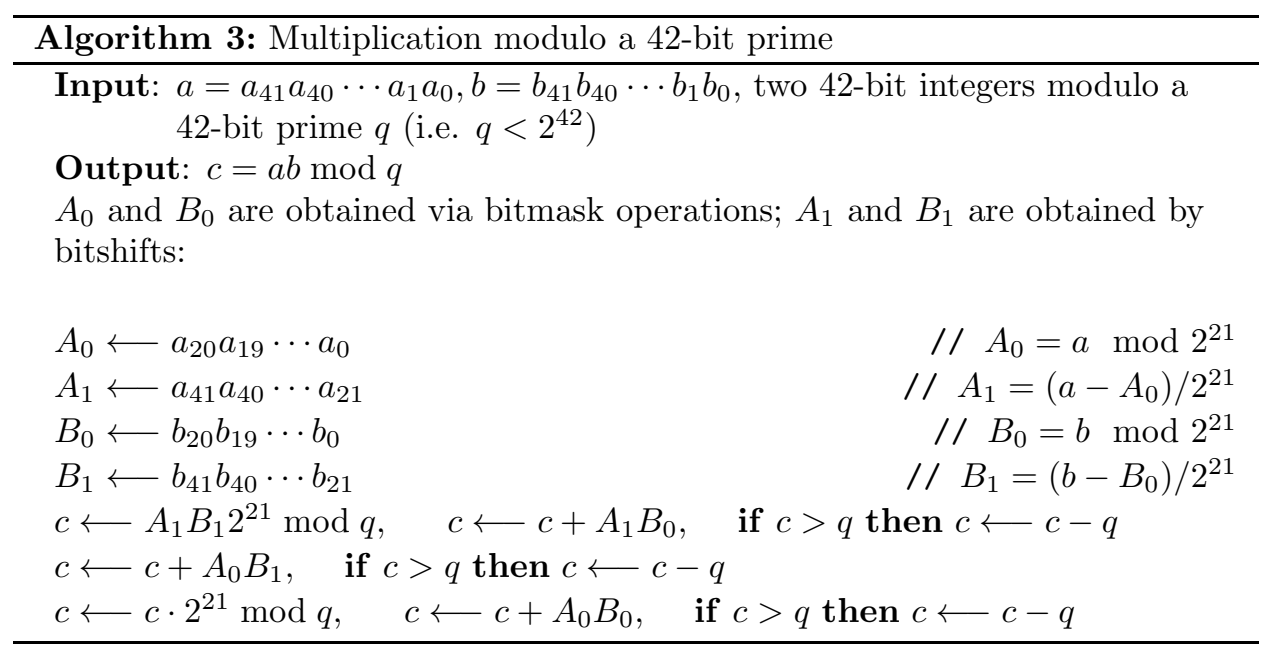

The FFT only gives us the coefficients of $\Phi_{n}(z)$ modulo a prime $q_{1}$. Our resulting polynomial, call it $H_{n}(z)$, will not equal $\Phi_{n}(z)$ if $A(n)>\frac{q_{1}}{2}$. We calculate $\Phi_{n}(z)$ modulo another prime $q_{2}$. We then reconstruct $H_{n}(z) \equiv \Phi_{n}(z) \bmod q_{1} q_{2}$ by Chinese remaindering. As such, we call this implementation the FFT-CRT algorithm. We do this with primes $q_{1}, q_{2} \ldots q_{l}$ until $\left\|H_{n}(z)\right\|_{\infty} \cdot 2^{20}<\frac{q_{1} q_{2} \ldots q_{l}}{2}$. We then take our solution $H_{n}(z)$ and use the FFT to test that

$$
H_{n}\left(\omega^{j}\right) \cdot \Phi_{m}\left(\omega^{j}\right)-\Phi_{m}\left(\omega^{j p}\right) \equiv 0 \bmod q_{l+1} \quad(0 \leq j<N),
$$


for some new prime $q_{l+1}$ with $N_{t h}$ primitive root $\omega$, where here $N$ is a power of two greater than $\phi(m) p$. This is because $H_{n}(z) \Phi_{m}(z)-\Phi_{m}\left(z^{p}\right)$ is potentially a polynomial of degree $\phi(m) p$. If equation (4) holds for all $j, H_{n}(z) \equiv \Phi_{n}(z)$ $\left(\bmod Q=q_{1} q_{2} \cdots q_{l} \cdot q_{l+1}\right)$. For $q_{l+1}>2^{40}$, it follows that all of the coefficients of $\Phi_{n}(z)$, modulo $Q$, lie in the interval $\left(\frac{-Q}{2^{60}}, \frac{Q}{2^{60}}\right)$. We consider 60 redundant bits sufficient. As we know, the cyclotomic coefficients roughly have a flat-bell distribution, it is very improbable that the height of $\Phi_{n}(z)$ is greater than $\frac{Q}{2}$ with all the coefficients strictly in the range $\left(\frac{-Q}{2^{60}}, \frac{Q}{2^{60}}\right)$ modulo $Q$. Indeed, all our results obtained by this method thus far have been consistent with results we have obtained by nonmodular algorithms. Table 1 lists the primes and the primitive roots we used in our computations.

TABLE 1. Primes and the primitive roots used in our FFT calculations

\begin{tabular}{|l|rlr|l|r|}
\hline & $q$ & $=$ & $r \cdot N+1$ & size of $q$ & $\omega$ \\
\hline$q_{1}$ & 2748779069441 & $=$ & $5 \cdot 2^{39}+1$ & $42 \mathrm{bits}$ & 243 \\
$q_{2}$ & 4123168604161 & $=$ & $15 \cdot 2^{38}+1$ & $42 \mathrm{bits}$ & 624392905782 \\
$q_{3}$ & 2061584302081 & $=$ & $15 \cdot 2^{37}+1$ & $41 \mathrm{bits}$ & 624392905781 \\
$q_{4}$ & 206158430209 & $=$ & $3 \cdot 2^{36}+1$ & $38 \mathrm{bits}$ & 10648 \\
$q_{5}$ & 2027224563713 & $=$ & $59 \cdot 2^{35}+1$ & $41 \mathrm{bits}$ & 1609812002788 \\
\hline
\end{tabular}

The brunt of the computation in our implementation of Table 1 takes place in the last division, as each successive division effectively increases the degree of the resulting intermediate polynomial by another factor. For squarefree $n$, we can compute $\Phi_{n}(z) \bmod q_{i}$ in $\mathcal{O}(N \cdot \log N)$ operations in $\mathbb{Z}_{q_{i}}$.

\section{Calculating $\Phi_{n}(z)$ as a quotient of sparse power series}

Every $n_{t h}$ root of unity is a primitive $d_{t h}$ root of unity for some unique $d \mid n$, and every $d_{t h}$ primitive root of unity is an $n_{t h}$ root of unity. Thus $z^{n}-1=\prod_{d \mid n} \Phi_{d}(z)$. Applying the Mőbius inversion formula to this, we obtain the well-known identity

$$
\Phi_{n}(z)=\prod_{d \mid n, d>0}\left(z^{d}-1\right)^{\mu\left(\frac{n}{d}\right)},
$$

where $\mu$ is the Möbius function. For $n>1$, the number of squarefree divisors of $n$ is even, in which case

$$
\Phi_{n}(z)=\prod_{d \mid n, d>0}\left(1-z^{d}\right)^{\mu\left(\frac{n}{d}\right)} .
$$

For example, for $n=105=3 \cdot 5 \cdot 7$,

$$
\Phi_{105}(z)=\frac{\left(1-z^{3}\right)\left(1-z^{5}\right)\left(1-z^{7}\right)\left(1-z^{105}\right)}{(1-z)\left(1-z^{15}\right)\left(1-z^{21}\right)\left(1-z^{35}\right)} .
$$

The sparseness of each term in this quotient lends itself to fast power series arithmetic. For the purposes of our algorithm, we treat $\Phi_{n}(z)$ as a truncated power series. Multiplying a power series $B(z)=\sum_{i=0}^{\infty} b(i) z^{i}$ by $1-z^{d}$ is easy:

$$
\left(\sum_{i=0}^{\infty} b(i) z^{i}\right)\left(1-z^{d}\right)=\sum_{i=0}^{d-1} b(i) z^{i}+\sum_{i=d}^{\infty}(b(i)-b(i-d)) z^{i}
$$


To divide by $1-z^{d}$ we merely multiply by the power series expansion of $\frac{1}{1-z^{d}}$ :

$$
\left(\sum_{i=0}^{\infty} b(i) z^{i}\right)\left(1+z^{d}+z^{2 d}+\cdots\right)=\sum_{i=0}^{\infty}(b(i)+b(i-d)+b(i-2 d)+\cdots) z^{i}
$$

Observe that the terms of $B(z)\left(1+z^{d}\right)$ and $B(z)\left(1+z^{d}\right)^{-1}$ depend strictly on terms of equal or lesser degree in $B(z)$. In addition, for $n>1$, the coefficients of $\Phi_{n}(z)$ are palindromic, that is, $a_{n}(k)=a_{n}(\phi(n)-k)$. So, to calculate the $\Phi_{n}(z)$ as a power series, we only need to compute and store the first $\frac{\phi(n)}{2}+1$ terms of $\Phi_{n}(z)$ and of any intermediate power series in our computation.

Division by $\left(1-z^{d}\right)$ done naively could be quadratic-time, ruining the efficiency of the algorithm. Suppose then that we have the coefficients $b(0), b(1), \ldots, b(D)$ of some power series $B(z)$ up to degree $D=\phi(n) / 2$, and we want to calculate $c(0), c(1), \ldots, c(D)$, the first $D+1$ coefficients of the power series

$$
C(z)=B(z) \cdot\left(1-z^{d}\right)^{-1}=\sum_{i=0}^{\infty} c(i)
$$

We can calculate all the $c(i)$ in linear time, without using additional memory to store intermediate results. By (7), $c(i)=b(i)$ for $0 \leq i<d$. For $i>d$, where $i=q d+r$ and $0 \leq r<i$,

$$
c(i)=b(i)+b(i-d)+\cdots+b(i-q d) .
$$

Since $c(i-d)=b(i-d)+b(i-2 d)+\cdots+b(i-q d)$, we have that

$$
c(i)=c(i-d)+b(i) .
$$

If we compute $c(i)=c(i-d)+b(i)$ for $i=d, d+1, \ldots, D$, we will have calculated all the $c(i)$ using at most one addition operation per term. Once we compute $c(i)$ we write that value over $b(i)$; we discard $B(z)$ as we compute $C(z)$. Algorithm 4 describes how we calculate $\Phi_{n}(z)$ using these techniques.

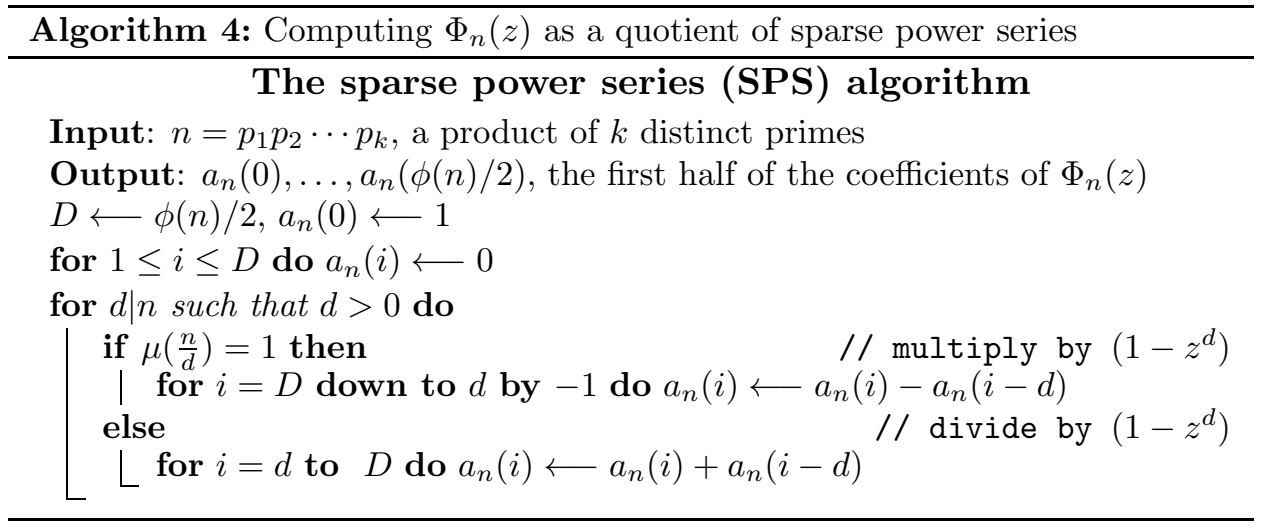

We call the algorithm the sparse power series (or SPS) algorithm. The SPS algorithm requires $\mathcal{O}\left(2^{k} \cdot \phi(n)\right)$ operations in $\mathbb{Z}$ to calculate $\Phi_{n}(z)$ of order $k$.

We have implemented 8-, 32-, 64-, and 128-bit versions of the SPS algorithm. A variant of our 32- and 64-bit versions are now used in Maple 14 and Sage. We do not use the GNU Multi-Precision library for multiprecision arithmetic. We handcoded our own 64- and 128-bit integer arithmetic that checks for overflow. This can be done without using redundant bits. We also have modular implementations 
of the SPS algorithm that calculates $\Phi_{n}(z)$ modulo 8-, 16- or 32-bit primes, and reconstructs $\Phi_{n}(z)$ by Chinese remaindering. This version is particularly useful for calculating cyclotomic polynomials that we cannot completely store in the main memory with large precision.

3.1. The sparse power series algorithm for inverse cyclotomic polynomials. Recall that $\Psi_{n}(z)$, the $n_{t h}$ inverse cyclotomic polynomial, is the monic polynomial whose roots are the $n_{t h}$ nonprimitive roots of unity. By (3), $\Phi_{n}(z) \cdot \Psi_{n}(z)=$ $z^{n}-1$. Thus we see from (6) that, for $n>1$,

$$
\Psi_{n}(z)=\frac{z^{n}-1}{\Phi_{n}(z)}=\left(z^{n}-1\right) \cdot \prod_{d \mid n}\left(1-z^{d}\right)^{-\mu\left(\frac{n}{d}\right)}=-\prod_{d \mid n, d<n}\left(1-z^{d}\right)^{-\mu\left(\frac{n}{d}\right)} .
$$

It becomes immediate that we can calculate $\Psi_{n}(z)$ in a manner ever-similar to the sparse power series algorithm for $\Phi_{n}(z)$. As with $\Phi_{n}(z)$, we need only compute the first half of the terms of $\Psi_{n}(z)$ because the coefficients of $\Psi_{n}(z)$ for $n>1$ are antipalindromic: given $\Psi_{n}(z)=c_{n}(0)+c_{n}(1) z+\cdots+c_{n}(n-\phi(n)) z^{n-\phi(n)}, n>1$, it holds that $c_{n}(k)=-c_{n}(n-\phi(n)-k)$. For squarefree $n=p_{1} p_{2} \ldots p_{k}$ the algorithm requires $\mathcal{O}\left(2^{k}(n-\phi(n))\right)=\mathcal{O}\left(2^{k} n\right)$ operations in $\mathbb{Z}$.

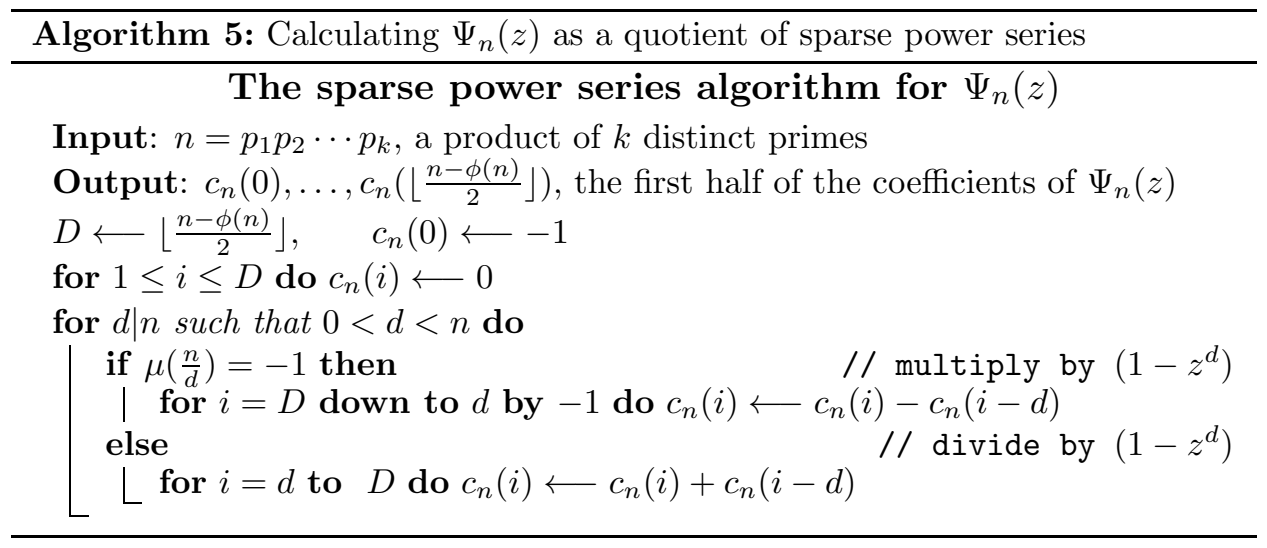

\section{A low-memory algorithm to calculate $A(n)$}

Calculating cyclotomic polynomials of very large degree using Algorithm 4 can bode problematic, as oftentimes $\Phi_{n}(z)$ will not fit in the main memory. In such a case, there are a variety of approaches to calculate $\Phi_{n}(z)$.

One approach is to calculate $\Phi_{n}(z)$ modulo primes $p_{i}$ sufficiently small such that we can fit $\Phi_{n}(z)$ into memory and write the images to hard disk. We then reconstruct the coefficients of $\Phi_{n}(z)$ sequentially from the images of $\Phi_{n}(z) \bmod p_{i}$. This minimizes the amount of computation we have to do on the hard disk.

For yet larger cyclotomic polynomials, we may not even be able to store the coefficients modulo a prime in memory. In which case we may be forced to write $\Phi_{n}(z)$ and our intermediate work to disk. This proves most costly, as the speed of the hard disk bottlenecks the sparse power series algorithm. As a motivating example, consider $\Phi_{n}(z)$ for

$$
n=2576062979535=3 \cdot 5 \cdot 29 \cdot 2609 \cdot 2269829 .
$$


This is the smallest $n=p_{1} p_{2} p_{3} p_{4} p_{5}$, a product of five distinct odd primes, such that

$$
p_{k} \equiv-1 \quad \bmod \prod_{i=1}^{k-1} p_{i} \text { for } k=2,3,4,5
$$

Nathan Kaplan 12 asked whether this cyclotomic polynomial is flat. To our knowledge, no one has yet found a flat cyclotomic polynomial of order 5 . This was a natural candidate to test for flatness. Kaplan [14] proved for $n=p_{1} p_{2} p_{3}$ satifying (8) for $k=2,3$ that $A(n)=1$. In addition, for every odd $n<3 \cdot 10^{8}$ of the form $n=p_{1} p_{2} p_{3} p_{4}$ satisfying (8) for $k=2,3,4$ is flat.

For our purposes, it is not necessary that we retrieve all the coefficients of $\Phi_{n}(z)$ at once, as we are mostly concerned with the height of $\Phi_{n}(z)$. Indeed, for a cyclotomic polynomial with degree in the tens of billions or beyond, there is very little we can feasibly do with $\Phi_{n}(z)$, so there may be no purpose to store it in memory for further computation.

Let $n=m p$ be a squarefree, odd integer with largest prime divisor $p$. We can compute $A(n)$ by inspecting some of the coefficients of $\Phi_{n}(z)$ sequentially such that we only have to store $m$ coefficients of $\Phi_{n}(z)$ at any one time. This algorithm takes $\mathcal{O}\left(m^{2}\right)=\mathcal{O}\left(\frac{n^{2}}{p^{2}}\right)$ integer operations, provided we have $\Phi_{m}(z)$ and $\Psi_{m}(z)$. Clearly, such an algorithm works best for $n$ with a large prime divisor, hence we affectionally call it the big prime algorithm.

Recall from (3) that $\Psi_{n}(z)=\left(z^{n}-1\right) / \Phi_{n}(z)$. By Lemma 3 ,

$$
\begin{aligned}
\Phi_{n}(z) & =\Phi_{m p}(z)=\frac{\Phi_{m}\left(z^{p}\right)}{\Phi_{m}(z)}=\Phi_{m}\left(z^{p}\right) \cdot \Psi_{m}(z) \cdot\left(z^{m}-1\right)^{-1}, \\
& =\Phi_{m}(z) \cdot \Psi_{m}(z) \cdot\left(-1-z^{m}-z^{2 m}-\cdots\right) .
\end{aligned}
$$

Write $\Phi_{m}(z)=\sum_{i=0}^{\phi(m)} b_{i} z^{i}$ and $\Psi_{m}(z)=\sum_{j=0}^{m-\phi(m)} c_{j} z^{j}$. From equation (9), we can express coefficients of $\Phi_{n}(z)=\sum_{k=0}^{\phi(n)} a_{n}(k) z^{k}$ in terms of the $b_{i}$ and $c_{j}$ :

$$
a_{n}(k)=-\sum_{\substack{i p+j \equiv k \bmod m \\ i p+j \leq k}} b_{i} c_{j} .
$$

This leads to the recurrence

$$
a_{n}(k)=a_{n}(k-m)-\sum_{i p+j=k} b_{i} c_{j},
$$

which is key to our algorithm. We formally describe the big prime algorithm in Algorithm 6

The algorithm iterates through pairs $(i, j)$ such that $0 \leq j \leq m-\phi(m)$, and $i p \leq \phi(n) / 2$. Since there are only $\mathcal{O}(m \cdot \phi(m))$ such pairs, it follows that the big prime algorithm takes $\mathcal{O}(m \cdot \phi(m)) \in \mathcal{O}\left(m^{2}\right)$ arithmetic operations. We only require the first half of the terms of $\Phi_{m}(z)$, as $\frac{\phi(m)}{2}>\frac{\phi(m)(p-1)}{2 p}=\frac{\phi(n)}{2 p}$.

We store the value of $a_{n}(k)$ in $\bar{a}(k \bmod m)$, and discard that value when computing $a_{n}(k+m)$. If $p>m-\phi(m)$, the degree of $\Psi_{m}(z)$, then Algorithm 6 does not consider every term of $\Phi_{n}(z)$ with degree less than $\phi(n) / 2$. In particular, if there is 


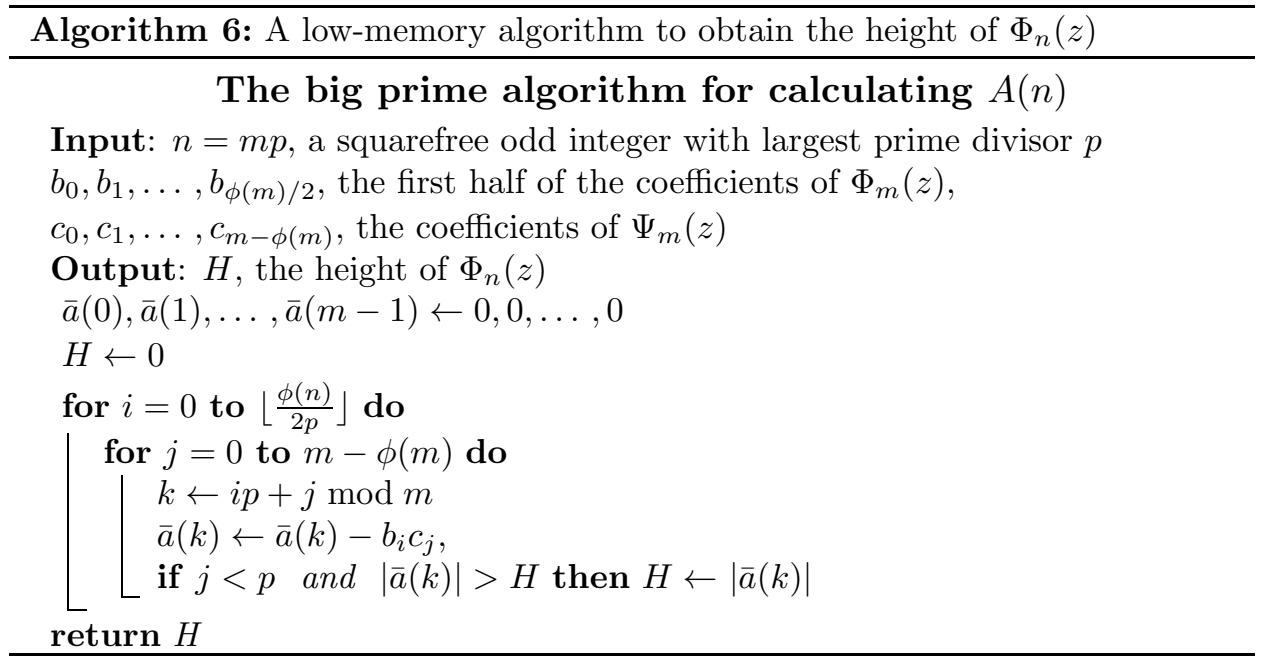

no pair $(i, j)$ such that $0 \leq i \leq \phi(m), 0 \leq j \leq m-\phi(n)$, and $i p+j=k$, then the big prime algorithm will not consider the term of the degree $k$. It follows from (10) that for such $k \geq m, a_{n}(k)=a_{n}(k-m)$, and for such $k<m, a_{n}(k)=0$. Thus we need not consider these terms to obtain the height $A(n)$.

It is easy to modify Algorithm 6 to generate all (or half) of the coefficients of $\Phi_{n}(z)$ (see Algorithm 7). In such a case the number of comparisons and arithmetic operations in $\mathbb{Z}$ increases from $\mathcal{O}(m \cdot \phi(m))$ to $\mathcal{O}(m \cdot \phi(m)+\phi(n))$. The computation space remains effectively the same; however, there is the additional $\mathcal{O}(\phi(n))$ space required to store the coefficients $a_{n}(k)$, whether to memory or disk.

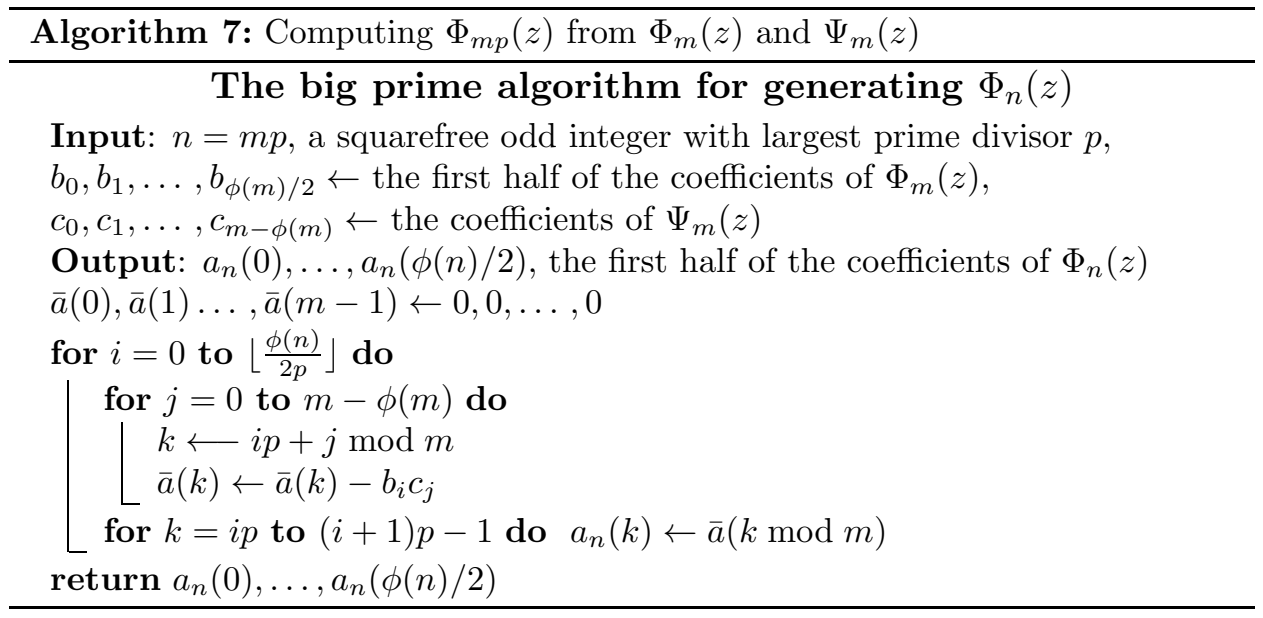

4.1. The big prime algorithm for $\Psi_{n}(z)$. Provided we have $\Phi_{m}(z)$ and $\Psi_{m}(z)$, we can generate the terms of $\Psi_{n}(z)$ for $n=m p$, in $\mathcal{O}(m \cdot \phi(m))$ arithmetic operations in $\mathbb{Z}$. To do this we calculate $\Psi_{n}(z)$ as a product of two polynomials. One can show that

$$
\Psi_{m p}(z)=\Phi_{m}(z) \Psi_{m}\left(z^{p}\right)
$$


by showing that both sides of (11) have the same roots. Thus if we again let $\Phi_{m}(z)=\sum_{i=0}^{\phi(m)} b_{i} z^{i}$ and $\Psi_{m}(z)=\sum_{j=0}^{m-\phi(m)} c_{j} z^{j}$, it is immediate that

$$
\Psi_{n}(z)=\sum_{i+p j=k} b_{i} c_{j} z^{k}
$$

where the sum is taken over $0 \leq i \leq \phi(m)$ and $0 \leq j \leq m-\phi(m)$. If $p>\phi(m)$, the implementation is especially simple, as we have at most one solution to $i+j p=k$ for a given integer $k$.

4.2. Implementation details and observations. The big prime algorithm and its variants were developed to calculate $A(n)$ for large squarefree $n$ with a large prime divisor. The $n$ for which we first implemented this algorithm were of the form $n=p_{1} p_{2} p_{3} p_{4} p_{5}$, a product of five distinct primes, such that $p_{k}>\prod_{i=0}^{k-1} p_{i}$ for $k=2,3,4,5$.

For such cases, to calculate $A(n)$ it is often advantageous to use a sparse representation for $\Phi_{m}(z)$ and $\Psi_{m}(z)$. For example, for $n=m p$ where $n=2576062979535$, $m=1134915$ and $p=2269829, \Phi_{m}(z)$ has degree 584192 but only 31679 terms, and $\Psi_{m}(z)$ has degree 550723 but only 2982 terms.

For yet larger examples of $n$, we cannot fit an array of $m$ integers in the main memory. For example, we wanted to see if $\Phi_{n}(z)$ was flat, where

$$
n=2876941641794034669918155=5 \cdot 29 \cdot 2029 \cdot 2353639 \cdot 4154714171969 .
$$

This is the smallest $n=p_{1} p_{2} p_{3} p_{4} p_{5}$ with $p_{1}=5$ that satisfies the set of congruences in (8). To test if $A(n)=1$ we first used the sparse power series algorithm to calculate $\Phi_{p_{1} p_{2} p_{3}}(z)$ and $\Psi_{p_{1} p_{2} p_{3}}(z)$, we then used the big prime algorithm to generate sparse representations of $\Psi_{m}(z)$ and $\Phi_{m}(z)$, where $m=p_{1} p_{2} p_{3} p_{4}$. We then calculated the terms of $\Phi_{n}(z)$ whose degrees were in a range modulo $m$ sufficiently small that we could fit into memory. We found that $\Phi_{n}(z)$ is not flat, as $\left|a_{n}(k)\right|=2$ for

$$
k=266298073621 \cdot 4154714171969+109596=184398730073579852543491,
$$

at which point we stopped the calculation.

\section{A COMPARISON OF THE EFFICIENCY OF THE ALGORITHMS}

Table 2 gives a comparison of the three algorithms to calculate $\Phi_{n}(z)$. For the purposes of this section, let $n$ be of the form

$$
n=p_{1} p_{2} \cdots p_{k}=m p
$$

a product of $k$ distinct primes with largest prime divisor $p$.

TABLE 2. Time and space complexities of the algorithms for $\Phi_{n}(z)$.

\begin{tabular}{|l|c|c|}
\hline \multicolumn{1}{|c|}{ Algorithm } & \# of operations in $\mathbb{Z}$ & space complexity \\
\hline \hline FFT-CRT (mod 1 prime, section 21) & $\mathcal{O}(n \log n)$ & $\mathcal{O}(\phi(n))$ \\
\hline Sparse power series (algorithm 4 & $\mathcal{O}\left(2^{k} \cdot \phi(n)\right)$ & $\mathcal{O}(\phi(n))$ \\
\hline Big prime (for $A(n)$ only, algorithm[6) & $\mathcal{O}(m \cdot \phi(m))$ & $\mathcal{O}(m)$ \\
\hline
\end{tabular}


The time and space complexities alone do not completely illuminate the advantages and disadvantages of each algorithm. Our implementation of the SPS algorithm (Algorithm 4) has several advantages over the FFT-CRT method (Algorithm 1). First, we can perform the calculations with little memory overhead; effectively all the memory used in the power series algorithm is to store the coefficients. The power series algorithm makes better use of the memory used to store terms. Using 64-bits of storage for one term gives us exactly 64-bit precision using the SPS method, whereas the FFT-CRT algorithm uses 64-bits to store a 42-bit terms. Most significantly, the arithmetic operations used in Algorithm 4 are strictly additions and subtractions, which take fewer CPU cycles than multiplication and division operations. In addition, the SPS algorithm exhibits better locality than the discrete Fast Fourier Transform. In practice, our implementation of the SPS algorithm is appreciably faster than that of the FFT-CRT algorithm (see Table 3).

TABLE 3. A comparison of running times of cyclotomic polynomial algorithms on a $3 \mathrm{GHz}$ Intel Xeon system

\begin{tabular}{|l|rrr|}
\hline \multicolumn{1}{|c|}{ Algorithm } & \multicolumn{3}{|c|}{ Running time to compute $\Phi_{n}(z)$ (seconds) } \\
\cline { 2 - 4 } & $n=255255$ & $n=1181895$ & $n=43730115$ \\
\hline Maple 13 cyclotomic command & 429.14 & - & - \\
\hline $\begin{array}{l}\text { Maple using an array of machine } \\
\text { precision integers }\end{array}$ & 13.78 & 197.37 & 371.93 \\
\hline $\begin{array}{l}\text { FFT-CRT using 2 42-bit primes, } \\
\text { plus a check prime (section [2) }\end{array}$ & 1.12 & 4.52 & 163.68 \\
\hline $\begin{array}{l}\text { FFT-CRT using 2 32-bit primes, } \\
\text { plus a check prime }\end{array}$ & 0.47 & 2.07 & 6.04 \\
\hline SPS, 64-bit (Algorithm 4) & 0.01 & 0.08 & 727.44 \\
\hline Big prime, 64-bit (Algorithm [6)
\end{tabular}

*Includes time to compute $\Phi_{m}(z)$ and $\Psi_{m}(z)$ via the 64-bit sparse power series algorithm

TABLE 4. Running time for the big prime algorithm (calculating height only, 8-bit version) on a $3 \mathrm{GHz}$ Intel Xeon system

\begin{tabular}{|r|r|r|}
\hline$n$ & factorization of $n$ & user time (seconds) \\
\hline 2576062979535 & $3 \cdot 5 \cdot 29 \cdot 2609 \cdot 2269829$ & 1.23 \\
36654908721735 & $3 \cdot 5 \cdot 29 \cdot 6959 \cdot 12108659$ & 3.29 \\
117714212390685 & $3 \cdot 5 \cdot 59 \cdot 3539 \cdot 37584179$ & 3.44 \\
1349266102959585 & $3 \cdot 5 \cdot 59 \cdot 8849 \cdot 172290029$ & 8.61 \\
16628239064490285 & $3 \cdot 5 \cdot 179 \cdot 10739 \cdot 576684299$ & 31.90 \\
\hline
\end{tabular}

We see that the big prime algorithm is relatively fast for $n=255255$ and 1181895 but considerably slower for $n=43730115$. This is what we expect, as the algorithm is quadratic-time. These examples, however, are not what the big prime algorithm was designed for. Table 4 shows timings used to calculate heights of $\Phi_{n}(z)$ for $n$ of the form discussed in Section 4.2. With the big prime algorithm, we can calculate heights of cyclotomic polynomials that may otherwise be infeasible to compute. 


\section{RESULTS}

All of our numerical results can be found in the appendix. All of the data we have computed is made available at

$$
\text { http://www.cecm.sfu.ca/ ada26/cyclotomic/ }
$$

6.1. Big heights and lengths of cyclotomic polynomials. To find cyclotomic polynomials with large heights, we needed bounds on $A(n)$. Bang [3] showed that for $n=p q$, a product of two primes, that $A(n)=1$; and for $n=p q r$, a product of three primes, that $A(n)<p$. Bloom [5] later proved for $n=p q r s$, a product of four primes with $p<q<r<s$, that $A(n)<p(p-1)(p q-1)$. Bateman, Pomerance, and Vaughan [4] proved a generalized albeit slightly weaker result: for $n=p_{1} p_{2} \cdots p_{k}$, a product of $k$ distinct primes with $p_{1}<p_{2}<\cdots<p_{k}$, we get

$$
A(n) \leq A\left(p_{1} p_{2} \cdots p_{k-1}\right) \prod_{j=0}^{k-2} S\left(p_{1} p_{2} \cdots p_{j}\right) .
$$

Using $S\left(p_{1} p_{2} \cdots p_{j}\right) \leq A\left(p_{1} p_{2} \cdots p_{j}\right) \cdot p_{1} p_{2} \cdots p_{j}$ and Bang's results, they inductively obtain that

$$
A\left(p_{1} p_{2} \cdots p_{k}\right) \leq \prod_{i=1}^{k-2} p_{i}^{2^{k-i-1}-1} .
$$

For example, $A\left(p_{1} p_{2} p_{3} p_{4} p_{5} p_{6}\right) \leq p_{1}^{15} p_{2}^{7} p_{3}^{3} p_{4}^{1}$. We use this bound to narrow our search for large values of $A(n)$, more specifically, $A(n)$ for which $A(n)>A(m)$ for $n<m$. Consider, for instance, $n=p_{1} p_{2} p_{3} p_{4} p_{5}$, a product of five primes where $p_{1}<p_{2}<p_{3}<p_{4}<p_{5}$. We have that $A(n)<p_{1}^{7} p_{2}^{3} p_{3}<n^{2.2}$. Given that $\max _{1 \leq k \leq n} A(k)$ exceeds $n^{2.2}$ for $n>43730115$, we can ignore products of five primes greater than 43730115 when searching for increasingly large heights.

Using the algorithms of Sections 2 and 3, we have created a library of data on $A(n)$ and $S(n)$. We include here our more noteworthy results. Table 6 shows those cyclotomic polynomials we have found whose height is greater than all those of smaller index. Excluding $n$ less than roughly 10000, those $n$ for which we obtain the largest heights also typically yield the largest lengths. Table 6 also gives $\log _{n}(A(n))$, which was of interest to us. Our results include the smallest $n$ such that $A(n)>n$, $A(n)>n^{2}, A(n)>n^{3}$, and $A(n)>n^{4}$. Table 5 shows $A(n)$ for $n$, a product of the $s$ smallest odd primes, for $1 \leq s \leq 9$.

Equation (12) suggests that if $A(n)$ is large, then $A(n p)$ is potentially large as well. This appears to be the case in Table 7 which shows computed values of $A(n)$ for which $A(n)>n^{4}$. Every $n$ in Table 7 is divisible by $m=40755=3 \cdot 5 \cdot 11 \cdot 13 \cdot 19$, which appears in Table [6 and has at least one of 29 or 37 as a prime factor. $40755 \cdot 29=1181895$ also appears in Table 6. In addition, every $n$ in Table 7 is neither divisible by 17, 23, nor (with the exception of $n=13162764615$ ) 7 .

Many of the examples in Table 7 are multiples of $m=43730115$, the first case such that $A(m)>m^{2}$. We mark these $n$ in Table 7 accordingly.

6.2. Computing $\Phi_{n}(z)$ of higher order. We currently cannot calculate $\Phi_{n}(z)$ of order greater than 9 . Calculating $\Phi_{n}(z)$ where $n=3 \cdot 5 \cdot 7 \cdot 11 \cdot 13 \cdot 17 \cdot 19 \cdot 23 \cdot 29 \cdot 31$, the product of the smallest ten odd primes, would require over 120 GB of memory to compute the polynomial using the SPS algorithm and 64-bit integers, and yet more memory using the FFT-CRT algorithm. 
We attempted to compute the cyclotomic polynomials for

$$
n=99660932085=3 \cdot 5 \cdot 11 \cdot 13 \cdot 19 \cdot 29 \cdot 37 \cdot 43 \cdot 53,
$$

which we expect to have a very large height. We computed $\Phi_{n}(z)$ modulo 32-bit primes by Algorithm 4, $\Phi_{n}(z)$ has degree 38041436160. Storing half the coefficients of $\Phi_{n}(z)$ with 32-bit precision requires roughly $76 \mathrm{~GB}$, and so the computation had to be done directly to disk. As such, the computation was particularly slow. We computed five images of $\Phi_{n}(z)$, after which the hard disk crashed. We will not be redoubling our efforts using this approach.

6.3. Flat cyclotomic polynomials. If $p$ is a prime, then $\Phi_{p}(z)=1+z+\cdots+z^{p-1}$ is trivially flat. All cyclotomic polynomials of order 2 are also flat. This is easy to verify using the following identity (see Lenstra, 11]). Given primes $p, q$, let $u, v$ be the integers such that $0<u<p, 0<v<q$, and $u q+v p=p q+1$. Then

$$
\Phi_{p q}(z)=\sum_{i=0}^{u-1} \sum_{j=0}^{v-1} z^{i q+j p}-\sum_{i=u}^{p-1} \sum_{j=v}^{q-1} z^{i q-j p-p q} .
$$

One can check that the degrees of the terms in each sum are distinct.

Cyclotomic polynomials of order three and greater are not, in general, flat.

6.3.1. Flat cyclotomic polynomials of order 3. There are $1566382 n<10^{8}$ of the form $n=p q r$, a product of three distinct odd primes, such that $A(n)=1$. Bachman 2] proved that $A(p q r)=1$ if $q \equiv-1 \bmod p$ and $r \equiv-1 \bmod p q$. Kaplan 14 proved a more general result, that $A(p q r)=1$ when $r \equiv \pm 1 \bmod p q$. For $n=p q r<10^{8}$, we find that $A(n)=1$ if $q \equiv 1 \bmod p$ and $r \equiv \pm 2 \bmod p q$. The aforementioned families account for 414832 of these flat cyclotomic polynomials of order 3.

6.3.2. Flat cyclotomic polynomials of order 4. Noe 22 has calculated flat cyclotomic polynomials of order 4 , for index $n<5 \cdot 10^{6}$. We extend his result to $n<3 \cdot 10^{8}$. There are 1389 such $n$, and each of these $n=p_{1} p_{2} p_{3} p_{4}$ satisfies

$$
p_{2} \equiv-1 \bmod p_{1}, \quad p_{3} \equiv \pm 1 \bmod p_{1} p_{2}, \quad p_{4} \equiv \pm 1 \bmod p_{1} p_{2} p_{3} .
$$

In addition, any cyclotomic polynomial $\Phi_{n}(z)$ of order four with $n=p_{1} p_{2} p_{3} p_{4}<$ $3 \cdot 10^{8}$ satisfying (14) is flat.

6.3.3. Are there flat cyclotomic polynomials of order 5 or greater? For $n<6.5 \cdot 10^{8}$, there is no cyclotomic polynomial $\Phi_{n}(z)$ of order 5 with height less than 4 . Table 8 shows the cyclotomic polynomials of order 5 and index $n$ such that is flatter than any cyclotomic polynomial of order 5 and smaller index, for $n<6.5 \cdot 10^{8}$. In an attempt to find a flat cyclotomic polynomial of order 5 , we computed $A(n)$ for $n$, a product of 5 distinct primes such that for $p$ dividing $n, n / p$ satisfies the set of congruences (14). That is, we computed $A(n)$ for $n=p_{1} p_{2} p_{3} p_{4} p_{5}$ satisfying

$$
\begin{aligned}
p_{2} \equiv-1 \bmod p_{1}, & p_{3} \equiv-1 \bmod p_{1} p_{2}, \\
p_{4} \equiv \pm 1 \bmod p_{1} p_{2} p_{3}, & p_{5} \equiv \pm 1 \bmod p_{1} p_{2} p_{3} p_{4} .
\end{aligned}
$$


We only consider $n$ satisfying (15) for which, given $\left(p_{1}, p_{2}, p_{3}, p_{4}\right), p_{5}$ is minimal for its congruence class modulo $p_{1} p_{2} p_{3} p_{4}$, because of the following theorem from Kaplan:

Theorem 5 (Kaplan, [13]). Let $m>0$ and let $p, q$ be primes such that $m<p<q$ and $p \equiv q(\bmod m)$. Then $A(m p)=A(m q)$.

We have calculated $A(n)$ for all such $n<2^{63}$. There are 5349 such $n$. Of these, $A(n)=2$ for 5212 cases and $A(n)=3$ for the remaining ones. We list the smallest indices $n$ of this form for which we have computed $A(n)=2$ in Table 9. The data for all 5349 cases can be found at our website.

6.4. Extrema of the $k_{t h}$ cyclotomic polynomial coefficient $a_{n}(k)$. Let $a(k)=$ $\max _{n}\left|a_{n}(k)\right|$, and let $a^{*}(k)=\max _{n} a_{n}(k)$ and $a_{*}(k)=\min _{n} a_{n}(k)$ be the one-sided bounds. We also define

$$
a^{* *}(k)=\max _{\text {squarefree } n} a_{n}(k) \quad \text { and } \quad a_{* *}(k)=\min _{\text {squarefree } n} a_{n}(k) .
$$

It is clear that $a^{* *}(k) \leq a^{*}(k)$ and $a_{* *}(k) \geq a_{*}(k)$.

Bachman [1] showed that for a constant $A_{0}$, and for sufficiently large $k$,

$$
\log a(k)=A_{0} \frac{\sqrt{k}}{(\log k)^{1 / 4}}\left(1+\mathcal{O}\left(\frac{\log \log k}{\sqrt{\log k}}\right)\right) .
$$

Gallot et al. [8] computed $a(k)$ for $k \leq 30$. We calculated $a(k)$ for $k \leq 172$ using a brute-force approach we detail below. Noe [21] calculated $a(k)$, for $k \leq 1000$ using a brute-force approach for $k \leq 128$, and a superior, fast method due to Grytczuk and Tropak [10] for larger $k$.

We verify his computation up to $k \leq 172$, and for those $k$ find the smallest index $n$ for which we obtain $\left|a_{n}(k)\right|=a(k)$. It is immediate from Algorithm 4 that $a_{n}(k)$ depends on the divisors of $n$ that are less than or equal to $k$. In particular, if $p$ and $q$ are distinct primes that are greater than $k$, then $a_{n}(k)=a_{n p q}(k)$ and $a_{n p}(k)=a_{n q}(k)$. Thus to calculate $a^{* *}(k)$, we need only consider $a_{n}(k)$ for $n$ of the form $n=m$ and $n=m q$, where $m$ is a product of distinct primes less than or equal to $k$, and $q$ is the first prime greater than $k$.

We used this brute-force approach to calculate $a^{* *}(k)$ and $a_{* *}(k)$ for $0 \leq k \leq 172$. This entailed inspection of $\Phi_{n}(z)$ for every squarefree $n$ that is a product of primes less than or equal to 173 , the $40_{\text {th }}$ prime. There are $2^{40}>10^{12}$ such $n$. We used a variant of Algorithm 4 to obtain the first 211 terms of $\Phi_{n}(z)$; instead of truncating the power series of $\Phi_{n}(z)$ to degree $\phi(n) / 2$, we truncate the power series to degree 210. In addition, given odd $n$, we use Lemma 1 to obtain the truncated power series of $\Phi_{2 n}(z)$. Since $\Phi_{2 n}(z)=\Phi_{n}(-z)$ for odd $n>1$, it follows that

$$
a_{2 n}(2 k)=a_{n}(2 k) \quad \text { and } \quad a_{2 n}(2 k+1)=-a_{n}(2 k+1) .
$$

Given $a_{n}^{* *}(d)$, for $0 \leq d \leq k$, one can obtain $a^{*}(k)$ by inspection. Suppose that $a^{*}(k)>a^{* *}(k)$, then there exists some nonsquarefree $n$ for which $a_{n}(k)>$ $a^{* *}(k)$. Write $n=m d$, where $m$ is the squarefree part of $n$. By Lemma 2 , 
$\Phi_{n}(z)=\Phi_{m}\left(z^{d}\right)$, and so if $d \mid k, a_{n}(k)=a_{m}(k / d)$, otherwise $a_{n}(k)=0$. Thus $a^{*}(k)=\max _{d \mid k} a^{* *}(k / d)$. Similarly, $a_{*}(k)=\min _{d \mid k} a_{* *}(k / d)$. Typically we find that $a^{*}(k)=a^{* *}(k) . k=118$ is the least $k>0$ such that $a(k)>k$, as $a^{*}(118)=$ 124 .

Another related problem is, given $b \in \mathbb{Z}$, find minimal $k$ such that there exists $n$ such that $a_{n}(k)=b$. Define

$$
\begin{aligned}
& \alpha(b)=\min _{a_{n}(k)=b} k \quad(\text { for } b \in \mathbb{Z}) \quad \text { and } \\
& \bar{\alpha}(b)=\min _{\left|a_{n}(k)\right|=b} k=\min (\alpha(b), \alpha(-b)) \quad(\text { for } b \geq 0),
\end{aligned}
$$

where the minima are taken over all pairs $(n, k)$ such that $n>0, k \geq 0$.

In our computation of $a(k)$ we have simultaneously computed $\alpha(b)$, for $-927 \leq$ $b \leq 927$, and the smallest $n$ for which $a_{n}(\alpha(b))=b$. Again by Lemma 2, we need only consider squarefree $n$ to computer $\alpha(b)$. Suppose $b \neq 0$ and $a_{n p^{2}}(k)=b$. Then, as $a_{n p^{2}}(k) \neq 0, p$ must divide $k$ by Lemma 2, and $a_{n p}(k / p)=b$. Thus $\alpha(b) \leq k / p<k$. Given that we know the maxima and minima of $a_{n}(k)$ for fixed $k \leq 172$, if the minimum $k$ we have found for which $\exists n \ni a_{n}(k)=b$ is less than or equal to 172 , then we know we have the exact value of $\alpha(b)$. The same holds if the minimum such $k$ equals 173 ; however, we cannot be certain that we have the smallest $n$ for which $a_{n}(173)=b$.

Table 10 shows $\bar{\alpha}(b)$ and least $n$ such that $\left|a_{n}(\bar{\alpha}(b))\right|=b$ for select values of $b$. We extend results by Bosma [6], and by Grytczuk and Tropak [10. Grytczuk and Tropak found results for $|b| \leq 10$. Bosma calculated results for $|b| \leq 50$. We have in fact calculated the smallest $k$ such that $a_{n}(k)=b$ for $-927 \leq b \leq 927$. All of these results can be found at our website.

\section{Appendix}

TABLE 5. $A(n)$ for $n$ a product of the smallest odd primes

\begin{tabular}{|r|c|r|r|}
\hline$n$ & factorization of $n$ & $A(n)$ & $\log _{n} A(n)$ \\
\hline 105 & $3 \cdot 5 \cdot 7$ & 2 & 0.148937 \\
1155 & $3 \cdot 5 \cdot 7 \cdot 11$ & 3 & 0.155791 \\
15015 & $3 \cdot 5 \cdot 7 \cdot 11 \cdot 13$ & 23 & 0.326043 \\
255255 & $3 \cdot 5 \cdot 7 \cdot 11 \cdot 13 \cdot 17$ & 532 & 0.504147 \\
4849845 & $3 \cdot 5 \cdot 7 \cdot 11 \cdot 13 \cdot 17 \cdot 19$ & $* 669606$ & 0.871381 \\
111546435 & $3 \cdot 5 \cdot 7 \cdot 11 \cdot 13 \cdot 17 \cdot 19 \cdot 23$ & 8161018310 & 1.231662 \\
3234846615 & $3 \cdot 5 \cdot 7 \cdot 11 \cdot 13 \cdot 17 \cdot 19 \cdot 23 \cdot 29$ & 2888582082500892851 & 1.941216 \\
\hline
\end{tabular}

*(Koshiba, 2002 [15]). 
TABLE $6 . n$ such that $A(n)>A(m)$ for $m<n$

\begin{tabular}{|c|c|c|}
\hline$n$ & $A(n)$ & $\log _{n} A(n)$ \\
\hline 1 & 1 & - \\
\hline 105 & 2 & 0.148937 \\
\hline 385 & 3 & 0.184540 \\
\hline 1365 & 4 & 0.192037 \\
\hline 1785 & 5 & 0.214959 \\
\hline 2805 & 6 & 0.225686 \\
\hline 3135 & 7 & 0.241716 \\
\hline 6545 & 9 & 0.250069 \\
\hline 10465 & 14 & 0.285125 \\
\hline 11305 & 23 & 0.335958 \\
\hline 17255 & 25 & 0.329943 \\
\hline 20615 & 27 & 0.331781 \\
\hline 26565 & 59 & 0.400255 \\
\hline 40755 & 359 & 0.554229 \\
\hline 106743 & 397 & 0.516829 \\
\hline 171717 & 434 & 0.503836 \\
\hline 255255 & 532 & 0.504147 \\
\hline 279565 & 1182 & 0.564147 \\
\hline 327845 & 31010 & 0.814317 \\
\hline 707455 & 35111 & 0.777039 \\
\hline 886445 & 44125 & 0.780927 \\
\hline 983535 & 59815 & 0.797093 \\
\hline 1181895 & 14102773 & 1.177309 \\
\hline 1752465 & 14703509 & 1.147954 \\
\hline 3949491 & 56938657 & 1.175678 \\
\hline 8070699 & 74989473 & 1.140162 \\
\hline 10163195 & 1376877780831 & 1.732388 \\
\hline 13441645 & 1475674234751 & 1.707101 \\
\hline 15069565 & 1666495909761 & 1.702652 \\
\hline 30489585 & 2201904353336 & 1.649191 \\
\hline 37495115 & 2286541988726 & 1.631796 \\
\hline 40324935 & 2699208408726 & 1.634490 \\
\hline 43730115 & 862550638890874931 & 2.347376 \\
\hline 169828113 & *31484567640915734941 & 2.369146 \\
\hline 185626077 & 42337944402802720258 & 2.373635 \\
\hline 416690995 & 80103182105128365570406901971 & 3.353164 \\
\hline 437017385 & 86711753206816303264095919005 & 3.349121 \\
\hline 712407185 & 111859370951526698803198257925 & 3.281324 \\
\hline 1250072985 & 137565800042644454188531306886 & 3.203113 \\
\hline 1311052155 & 192892314415997583551731009410 & 3.211947 \\
\hline 1880394945 & 64540997036010911566826446181523888971563 & 4.400339 \\
\hline 2317696095 & 67075962666923019823602030663153118803367 & 4.359458 \\
\hline 13162764615 & **5465808676670557863536977958031695430428633 & 4.223361 \\
\hline
\end{tabular}

*First instance such that $A(n)>2^{64}$.

${ }^{* *}$ We have yet to show that $A(13162764615)>A(m)$ for all $m<13162764615$. 
TABLE 7. $n$ such that $A(n)>n^{4}$

\begin{tabular}{|r|r|r|}
\hline$n$ & $A(n)$ & $\log _{n} A(n)$ \\
\hline$* 1880394945$ & 64540997036010911566826446181523888971563 & 4.400339 \\
$*_{2} 2317696095$ & 67075962666923019823602030663153118803367 & 4.359458 \\
$* 2580076785$ & 44178992295210157476612718521873077815356 & 4.318615 \\
$* 2667537015$ & 50978590999382303149290759045486931349075 & 4.318577 \\
2693538705 & 41311315644168801150352807111933599181273 & 4.306965 \\
$* 2929917705$ & 38986521325602066434123587685733770107831 & 4.287687 \\
2998467615 & 50703798374791014119206172479361263858737 & 4.295185 \\
3100110585 & 37023124240370602498540690923261030138801 & 4.274245 \\
3405039495 & 41344572168478125565953675666880875630891 & 4.261005 \\
3436583865 & 29420710476505598428925342851511436269885 & 4.243720 \\
$* 3629599545$ & 31146417203818726557508589352280096726851 & 4.235775 \\
3695785665 & 27544659617064926303551956412846199778051 & 4.226722 \\
3821066535 & 31128120202581145792970998453196458758768 & 4.225879 \\
3825631095 & 40071361165347422625605753503633105870103 & 4.237096 \\
3955313505 & 27667056853522527517062927200210380189261 & 4.213942 \\
4196909145 & 24175660733238789429230720957313695678993 & 4.196578 \\
4218183255 & 31889907167996287439150048606465589433551 & 4.208117 \\
4253640105 & 24726477535222303105776318525136248038167 & 4.195053 \\
4344360735 & 32507457200647115397398921638675751619003 & 4.203392 \\
$* 4416741615$ & 30381795842095831309232742046965986663843 & 4.197220 \\
4672030935 & 22760422406095676210121193519495242514072 & 4.173655 \\
$* 4679122305$ & 24693521216952698497060423547227286104011 & 4.177032 \\
4715312745 & 18998617883089513487054213546426519137567 & 4.163816 \\
$* 4766582535$ & 27232450597992733695751246276504205431221 & 4.177952 \\
$* 13162764615$ & 5465808676670557863536977958031695430428633 & 4.223361 \\
\hline
\end{tabular}

* multiple of 43730115

TABLE 8. $\Phi_{n}(z)$ of order 5 that are flatter than all $\Phi_{m}(z)$ of order 5 for $m<n$, for $n \leq 6.5 \cdot 10^{8}$

\begin{tabular}{|r|r|r|}
\hline$n$ & factorization of $n$ & $A(n)$ \\
\hline 15015 & $3 \cdot 5 \cdot 7 \cdot 11 \cdot 13$ & 23 \\
23205 & $3 \cdot 5 \cdot 7 \cdot 13 \cdot 17$ & 21 \\
31395 & $3 \cdot 5 \cdot 7 \cdot 13 \cdot 23$ & 15 \\
574665 & $3 \cdot 5 \cdot 7 \cdot 13 \cdot 421$ & 14 \\
774795 & $3 \cdot 5 \cdot 7 \cdot 47 \cdot 157$ & 13 \\
1331715 & $3 \cdot 5 \cdot 7 \cdot 11 \cdot 1153$ & 12 \\
2666895 & $3 \cdot 5 \cdot 7 \cdot 11 \cdot 2309$ & 9 \\
3725085 & $3 \cdot 5 \cdot 7 \cdot 13 \cdot 2729$ & 7 \\
40765935 & $3 \cdot 5 \cdot 7 \cdot 43 \cdot 9029$ & 6 \\
48713385 & $3 \cdot 5 \cdot 7 \cdot 47 \cdot 9871$ & 5 \\
76762245 & $3 \cdot 5 \cdot 7 \cdot 59 \cdot 12391$ & 4 \\
\hline
\end{tabular}


TABLE $9 . \Phi_{n}(z)$ of order 5 such that $A(n)=2$

\begin{tabular}{|r|r|}
\hline$n$ & factorization of $n$ \\
\hline 1147113361785 & $3 \cdot 5 \cdot 29 \cdot 1741 \cdot 1514671$ \\
2294224451565 & $3 \cdot 5 \cdot 29 \cdot 1741 \cdot 3029339$ \\
2576062979535 & $3 \cdot 5 \cdot 29 \cdot 2609 \cdot 2269829$ \\
7157926096635 & $3 \cdot 5 \cdot 29 \cdot 4349 \cdot 3783629$ \\
7157929880265 & $3 \cdot 5 \cdot 29 \cdot 4349 \cdot 3783631$ \\
14031384951165 & $3 \cdot 5 \cdot 29 \cdot 6089 \cdot 5297431$ \\
15456385821615 & $3 \cdot 5 \cdot 29 \cdot 2609 \cdot 13618981$ \\
36654908721735 & $3 \cdot 5 \cdot 29 \cdot 6959 \cdot 12108659$ \\
39282436838685 & $3 \cdot 5 \cdot 59 \cdot 3541 \cdot 12535141$ \\
44151142013985 & $3 \cdot 5 \cdot 59 \cdot 5309 \cdot 9396929$ \\
44151151410915 & $3 \cdot 5 \cdot 59 \cdot 5309 \cdot 9396931$ \\
46392857518515 & $3 \cdot 5 \cdot 29 \cdot 7829 \cdot 13622461$ \\
\hline
\end{tabular}

TABLE $10 . \bar{\alpha}(b)$, the least $k$ for which $b$ occurs as $\left|a_{n}(k)\right|$; and the least $n$ for which $\left|a_{n}(\bar{\alpha}(k))\right|=b$; for select $b \leq 927$

\begin{tabular}{|c|c|c|}
\hline$a$ & $\bar{\alpha}(b)$ & $n$ \\
\hline 0 & 1 & 4 \\
\hline 1 & 0 & 1 \\
\hline 2 & 7 & 105 \\
\hline 3 & 17 & 323323 \\
\hline 4 & 23 & 1062347 \\
\hline 5 & 30 & 37182145 \\
\hline 6 & 36 & 215656441 \\
\hline 7 & 43 & 65552121635 \\
\hline 8 & 46 & 845904650955 \\
\hline 9 & 47 & 75145115045 \\
\hline 10 & 52 & 30704573184285 \\
\hline 20 & 70 & 152125131763605 \\
\hline 30 & 82 & 307444891294245705 \\
\hline 40 & 89 & 1352450076803386856295 \\
\hline 50 & 95 & 3929160775540133527939545 \\
\hline 60 & 99 & 194825753248734022710250308207855 \\
\hline 70 & 100 & 163957329252276946718326137628485 \\
\hline 80 & 106 & 4281025919618354440889355 \\
\hline 90 & 106 & 1200321465765450313917852148868594655 \\
\hline 100 & 112 & 23806785138997669045785703155 \\
\hline 200 & 132 & 162938425981534060763635083977029188109663335 \\
\hline 300 & 143 & 2326975571029326286598990252532796074781464457755 \\
\hline 400 & 153 & 5412131370764127757636390017210695 \\
\hline 500 & 158 & 5921057432644596149106845426292101971454108035 \\
\hline 600 & 163 & 50967866897743398269290966947741571435 \\
\hline 700 & 167 & 41211036991280460777846257111155083155 \\
\hline 800 & 170 & 523848308647668419809505921108741216619845 \\
\hline 900 & 173 & *480644324429304014052020896687401734836765 \\
\hline 927 & 173 & *1269140374116844321897058519227927779943780451272073121291475705 \\
\hline
\end{tabular}

*There may exist smaller $n$ for which $\left|a_{n}(k)\right|=b$. 


\section{REFERENCES}

[1] G. Bachman. On the coefficients of cyclotomic polynomials. Mem. Amer. Math. Soc., 106(510):vi+80, 1993. MR.1172916 (94d:11068)

[2] G. Bachman. Flat cyclotomic polynomials of order three. Bull. London Math. Soc., 38(1):5360, 2006. MR 2201603 (2006j:11032)

[3] A. S. Bang. Om ligningen $\phi_{n}(x)=0$. Nyt Tidsskrift for Mathematik, (6):6-12, 1895.

[4] P.T. Bateman, C. Pomerance, and R.C. Vaughan. On the size of the coefficients of the cyclotomic polynomial. In Topics in classical number theory, Vol. I, II (Budapest, 1981), volume 34 of Colloq. Math. Soc. János Bolyai, pages 171-202. North-Holland, Amsterdam, 1984. MR781138 (86e:11089)

[5] D.M. Bloom. On the coefficients of the cyclotomic polynomials. Amer. Math. Monthly, 75:372-377, 1968. MR0227086 (37:2671)

[6] W. Bosma. Computation of cyclotomic polynomials with Magma. In Computational algebra and number theory (Sydney, 1992), volume 325 of Math. Appl., pages 213-225. Kluwer Acad. Publ., Dordrecht, 1995. MR1344932 (96j:11142)

[7] Paul Erdös. On the coefficients of the cyclotomic polynomial. Bull. Amer. Math. Soc., 52:179184, 1946. MR0014110 (7:242e)

[8] Y. Gallot, P. Moree, and H. Hommerson. Value distribution of cyclotomic polynomial coefficients. Available at http://arxiv.org/abs/0803.2483.

[9] K.O. Geddes, S.R. Czapor, and G. Labahn. Algorithms for Computer Algebra. Kluwer Academic Publishers, Boston, 1992. MR1256483 (96a:68049)

[10] A. Grytczuk and B. Tropak. A numerical method for the determination of the cyclotomic polynomial coefficients. In Computational number theory (Debrecen, 1989), pages 15-19. de Gruyter, Berlin, 1991. MR 1151850 (93c:11116)

[11] Jr H.W. Lenstra. Vanishing sums of roots of unity. In Proceedings, Bicentennial Congress Wiskundig Genootschap (Vrije Univ., Amsterdam, 1978), Part II, volume 101 of Math. Centre Tracts, pages 249-268, Amsterdam, 1979. Math. Centrum. MR.541398 (81c:10044)

[12] N. Kaplan. Personal correspondence.

[13] N. Kaplan. Flat cyclotomic polynomials of order four or higher. Integers, 10:A30, 357-363, 2010. MR2684126

[14] N. Kaplan. Flat cyclotomic polynomials of order three. J. Number Theory, 127(1):118-126, 2007. MR2351667 (2008k:11031)

[15] Y. Koshiba. On the calculations of the coefficients of the cyclotomic polynomials. Rep. Fac. Sci. Kagoshima Univ., (31):31-44, 1998. MR1718402 (2000g:11126)

[16] Y. Koshiba. On the calculations of the coefficients of the cyclotomic polynomials. II. Rep. Fac. Sci. Kagoshima Univ., (33):55-59, 2000. MR1833785 (2002i:11132)

[17] H. Maier. The size of the coefficients of cyclotomic polynomials. In Analytic number theory, Vol. 2 (Allerton Park, IL, 1995), volume 139 of Progr. Math., pages 633-639. Birkhäuser Boston, Boston, MA, 1996. MR1409383 (97g:11106)

[18] H.L. Montgomery and R. C. Vaughan. The order of magnitude of the $m$ th coefficients of cyclotomic polynomials. Glasgow Math. J., 27:143-159, 1985. MR819835(87e:11026)

[19] P. Moree. Inverse cyclotomic polynomials. J. Number Theory, 129(3):667-680, 2009. MR2488596(2009k:11199)

[20] T.D. Noe. Least $k$ such that the $x^{n}$ coefficient of cyclotomic polynomial phi(k,x) has the largest possible magnitude. Sequence A138475 in N. J. A. Sloane (Ed.), The OnLine Encyclopedia of Integer Sequences (2008), http://www.research.att.com/ njas/ sequences/A138475.

[21] T.D. Noe. Maximum possible magnitude of the $x^{n}$ coefficient of a cyclotomic polynomial. Sequence A138474 in N. J. A. Sloane (Ed.), The On-Line Encyclopedia of Integer Sequences (2008), published electronically at http://www.research.att.com/ njas/ sequences/A138474.

[22] T.D. Noe. Numbers $\mathrm{n}$ such that $\mathrm{phi}(\mathrm{n}, \mathrm{x})$ is a flat cyclotomic polynomial of order four. Sequence A117318 in N. J. A. Sloane (Ed.), The On-Line Encyclopedia of Integer Sequences (2008), http://www.research.att.com/〜njas/sequences/A117318. 
[23] T.D. Noe. Numbers $n$ such that $\operatorname{phi}(\mathrm{n}, \mathrm{x})$ is a flat cyclotomic polynomial of order three. Sequence A117223 in N. J. A. Sloane (Ed.), The On-Line Encyclopedia of Integer Sequences (2008), http://www.research.att.com/ njas/sequences/A117223.

[24] T.D. Noe. Odd squarefree numbers $n$ such that the cyclotomic polynomial phi(n,x) is not coefficient convex. Sequence A146960 in N. J. A. Sloane (Ed.), The On-Line Encyclopedia of Integer Sequences (2008), http://www.research.att.com/ njas/sequences/A146960.

[25] R. Thangadurai. On the coefficients of cyclotomic polynomials. In Cyclotomic fields and related topics (Pune, 1999), pages 311-322. Bhaskaracharya Pratishthana, Pune, 2000. MR1802391 (2001k:11213)

[26] J. von zur Gathen and J. Gerhard. Modern computer algebra. Cambridge University Press, Cambridge, second edition, 2003. MR2001757 (2004g:68202)

Centre for Experimental and Constructive Mathematics, Simon Fraser University, Burnaby, BC V5A 1S6, CANada

E-mail address: ada26@sfu.ca

Centre for Experimental and Constructive Mathematics, Simon Fraser University, Burnaby, BC V5A 1S6, Canada

E-mail address: mmonagan@cecm.sfu.ca 